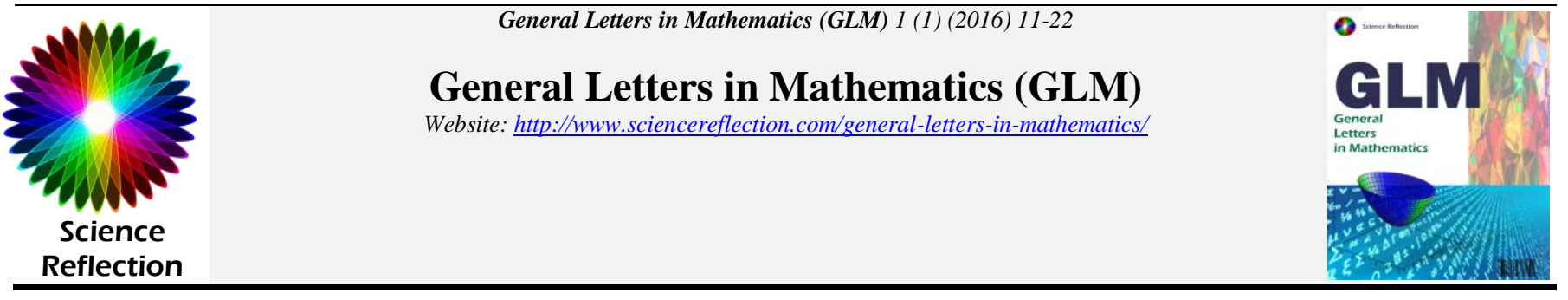

\title{
Congruences on Inverse Semigroups using Kernel Normal System
}

\author{
Laila M.Tunsi \\ University of Tripoli, Department of Mathematics, Tripoli, Libya \\ Laila.tunsi@gmail.com
}

\begin{abstract}
Congruences on inverse semigroups via the (kernel-trace) method introduced by Scheiblich in 1974. In this paper we discuss the congruences on inverse semigroups by using the technique of Kernel normal systems. Congrueneses on inverse semigroup were described in terms of congruences pairs $\left(\operatorname{ker}^{\rho_{s}} \operatorname{tr}{ }^{\rho}\right)$. It is natural to ask if this strategy can be extended to include regular semigroups. Feigenbaum in 1979 has achieved this. However, this approach has not proved to be the best possible for congruences on regular semigroups in general. Whilst it is possible to describe abstractly the trace and kernel of congruence on a regular semigroup, these descriptions are unwieldy. The technique which has proved most useful for studying congruences on arbitrary regular semigroups is that due to Preston of Kernel normal systems.
\end{abstract}

\section{Indexing terms/Keywords}

Inverse semigroup, congruence, Kernel, regular semigroup, Kernel normal systems.

\section{Introduction}

Congruence on an algebraic is an equivalence relation on the structure which is compatible with all the operations on the structure. The compatibility condition allows corresponding operations to be defined on the equivalence classes of a congruence to obtain a similar structure. All homomorphic images of an algebraic structure arise in this way and consequently, a great deal of effort has been put into studying congruences.

Semigroups provide one of the simplest types of structure for which congruences are not determined by just one equivalence class. In Section 2 we present a number of elementary concepts and propositions on semigroups. In Section 3 we describe the Kernel (or Kernel normal system) of a congruence on an inverse semigroups. The use of the capital $\mathrm{K}$ is to distinguish the Kernel of a congruence from its kernel defined in Section 2.

The first characterization of arbitrary congruences was due to Preston [7] who introduced the notion of a Kernel normal system. Later approaches characterized congruences on an inverse semigroup in terms of 
restrictions to the set of idempotents and the kernels of the congruences, the kernel being the set of elements related to idempotents. This culminated in Petrich's theory of congruence pairs [6]. In [2] Meakin gave an alternative definition for a Kernel normal system on an orthodox semigroup.

We give a full account of Kernel normal systems in Section 4. In particular, we include the result that a congruence on an inverse semigroup is completely determined by a Kernel normal system. The final set of conditions is equivalent to the first set, in fact, a combination of Exercises 6 and 8 on p.63 of [1].

\section{Introductory concepts}

In this section we shall define some of the basic concepts which will be used throughout the paper.

A relation $\rho$ on a semigroup $S$ is called right compatible if For all $a, b, c, \in S \quad[(a, b) \in \rho \Rightarrow(a c, b c) \in \rho]$ and $\rho$ is called left compatible if For all $a, b, c \in S \quad[(a, b) \in \rho \Rightarrow(c a, c b) \in \rho]$.

A right (left) congruence on a semigroup $S$ is defined to be an equivalence relation $\rho$ on $S$ which is right (left) compatible. A relation $\rho$ on $S$ which is both a right and a left congruence is called a congruence on $S$.

Proposition 2.1 An equivalence relation $\rho$ on a semigroup $S$ is a congruence if and only if for any $a, b, c, d \in S \quad(a, b) \in \rho,(c, d) \in \rho \Rightarrow(a c, b d) \in \rho$.

Given a congruence $\rho$ on a semigroup $S$, note that

$$
x \rho=\left\{x^{\prime} \in S: x \rho x^{n}\right\}
$$

We refer to $x \rho$ as the congruence class of an element $x \in S$. One defines a semigroup structure on the set $S / \rho$ of all congruence classes of $S$ by taking $x \rho . y \rho=(x y) \rho$ for any $x, y \in S$. This operation on $S / \rho$ is welldefined and it is easily seen to be associative and so $\left(S / \rho_{,}\right)$is a semigroup.

Now the proof of the following fundamental theorem of homomorphisms is straightforward.

Theorem 2.1 Let $\rho$ be a congruence on a semigroup $S$. Then $S / \rho$ is a semigroup and the mapping $\rho^{ \pm}: S \rightarrow S / \rho$ defined by $x \rho^{ \pm}=x \rho$ for all $x \in S$ is a homomorphism with ker $\rho^{ \pm}=\rho$.

Theorem 2.2 Let $\varphi: S \rightarrow T$ be a semigroup homomorphism. Then

$$
\operatorname{ker} \varphi=\varphi \circ \varphi^{-1}=\{(a, b) \in S \times S: a \varphi=b \varphi\}
$$

is a congruence on $S$ and there is a homomorphism $\varphi^{\prime}=S / \operatorname{ker} \varphi \rightarrow T \operatorname{such}$ that $\operatorname{im} \varphi^{\prime}=\operatorname{im} \varphi$ and $(\operatorname{ker} \varphi)^{ \pm}$ $. \varphi^{\prime}=\varphi$.

Definition 2.1 Equivalence relations $\mathcal{L}$ and $R$ on $S$ defined by the rule that $a \mathcal{L} b(a \mathcal{R} b)$ if and only if a and $b$ generate the same principal left (right) ideal. $D$ is the smallest equivalence relation containing $\mathcal{L}$ and $\mathcal{R}$.

These relations called Green's relations [2]. 
Lemma 2.1 Let $a$ and $b$ be elements of a semigroup $S$. Then $a \mathcal{L} b$ if and only if there exist $x, y$ in $S^{1}$ such that $x a=b$ and $y b=a$. Dually, $a \mathcal{R} b$ if and only if there exist $u, v$ in $S^{l}$ such that $a u=b$ and $b v=a$.

Proposition 2.2 The relations $\mathcal{L}$ and $\mathcal{R}$ commute.

Definition 2.2 An element a of a semigroup $S$ is called regular if there exists an element $x$ in $S$ such that $a x a=a$. A semigroup $S$ is called regular if all its elements are regular.

Definition 2.3 A semigroup $S$ is called an inverse semigroup if every a in $S$ possesses a unique inverse, that is, there exists a unique element $a^{-1}$ in $S$ such that

$$
a a^{-1} a=a \text { and } a^{-1} a a^{-1}=a \text {. }
$$

Such a semigroup is certainly regular and $|V(a)|=1$ for all $a \in S$. In this case we write $V(a)=\left\{a^{-1}\right\}$, so that $a^{-1}$ is the unique inverse of $a$.

Theorem 2.3 Let $\mathrm{S}$ be a semigroup. The following statements are equivalent:

- $\quad S$ is an inverse semigroup;

- $\quad S$ is regular and its idempotents commute;

- Each $\mathcal{L}$-class and each $\mathcal{R}$-class of $S$ contains a unique idempotent;

- Each principal left ideal and each principal right ideal of S contains a unique idempotent generator. The proof of the following proposition follows from the definition.

Proposition 2.3 Let S be an inverse semigroup with semilattice of idempotent $E$. Then we have

a) $\left(a^{-1}\right)^{-1}=$ a for every $a \in S$.

b) $e^{-1}=$ efor every $e \in E$.

c) $(a b)^{-1}=b^{-1} a^{-1}$ for every $a, b \in S$.

d) $a e a^{-1} \in E, a^{-1} e a \in E$ for every $a \in S$ and $e \in E$.

e) $a \mathcal{R} b$ if and only if $a a^{-1}=b b^{-1}, a \mathcal{L} b$ if and only if $a^{-1} a=b^{-1} b$.

f) If $e_{3} f \in E$, then $e D f \in S$ if and only if there exists $a$ in $S$ such that $a a^{-1}=e$ and $a^{-1} a=f$.

\section{Kernel normal systems}

Let $\rho$ be a congruence on a regular semigroup $S$. The Kernel normal system associated with $\rho$, or more briefly, Kernel of $\rho(\operatorname{Ker} \rho)$ is defined by

$$
\operatorname{Ker} \rho=\{e \rho: e \in E(S)\}
$$

One also sees Ker $\rho$ defined as

$$
\operatorname{Ker} \rho=\{\rho: a \rho \in E(S / \rho)\}
$$


Since $\mathrm{S}$ is regular, these are the same, by [3]. The reader should be careful not to confuse the concepts of ker $\rho$ and $\operatorname{Ker} \rho$. By definition we have

$$
\operatorname{ker} \rho=U\{A: A \in \operatorname{Ker} \rho\} .
$$

The set $A=\left\{A_{i}: i \in I\right\}$ of subsets of a regular semigroup $S$ is defined to be a Kernal normal system of $S$ if A satisfies

$\left.\mathrm{C}_{1}\right) A_{i} \cap A_{j}=\varphi$ if $i \neq j$

$\mathrm{C}_{2}$ ) each $A_{i}$ contains an idempotent of $S$ and each idempotent of $S$ is contained in some $A_{i}$

$\left.\mathrm{C}_{3}\right) x A_{i} y \cap A_{j} \neq \varphi$ implies $x A_{i} y \subseteq A_{j}$ for each $x, y \in S^{1}$ and $i, j \in I$.

We aim to show that the set of Kernel normal systems of $\mathrm{S}$ is exactly the set of Kernels of congruences on $\mathrm{S}$, and further, every congruence on S determined by its Kernel.

The Kernel normal systems can be defined also for inverse semigroups as we will show in the next section.

Lemma 3.1 Let $\rho$ be a congruence on a regular semigroup $S$. Then the Kernel of $\rho$ is a Kernel normal system of S.

Proof Let $A=\left\{A_{i}=e \rho ; i \in I\right\}$ be the Kernel of $\rho$. We have to show that $A$ satisfies conditions $\mathrm{C}_{1}, \mathrm{C}_{2}$ and $\mathrm{C}_{3}$. Condition $\mathrm{C}_{1}$ is clear since the $A_{\mathrm{i}}$ 's are congruence classes. Condition $\mathrm{C}_{2}$ is immediate. To show condition $\mathrm{C}_{3}$, suppose that $x A_{i} y \cap A_{j} \neq \varphi$, for some $x, y \in S^{1}$ and $i, j \in I$. Then there exist $a \in A_{i}, b \in A_{j}$ with $x a y=b$. Let $c \in A_{i}$ and consider $x c y$. Since $A_{i}$ and $A_{\mathrm{j}}$ are congruence classes, we have $x c y \rho x a y=b$, so that $x c y \in A_{j}$. Thus $x A_{i} y \subseteq A_{j}$.

Theorem 3.1 Two congruences $\sigma$ and $\rho$ on a regular semigroup $S$ coincide if and only if they have the same Kernel.

Proof Let $\rho$ and $\sigma$ be two congruences on a regular semigroup $S$ having the same Kernel. We have to show that $\rho=\sigma$. Let $(x, y) \in \sigma$. Since $S$ is regular, there exist $a, b$ in $S$ such that $x a x=x, \quad a x a=a, \quad y b y=y \quad$ and $\quad b y b=b$, so that $x a, b y$ are idempotents of $S$. Since $\sigma$ is right compatible, $(x a, y a) \in \sigma_{x}$ and from left compatibility we get $(b x, b y) \in \sigma$ and because $\rho$ and $\sigma$ have the same Kernel, and $x a$, by are idempotents, $(x a, y a) \epsilon \rho$ and $(b x, b y) \epsilon \rho$.

Now $x=x a x \rho y a x=y b y a x \rho y b x a x=y b x \rho y b y=y$. Then $x \rho y$ that is, $(x, y) \in \rho$ which implies that $\sigma \subseteq \rho$. The argument can be repeated with $\sigma$ and $\rho$ interchanged giving $\rho \subseteq \sigma$ whence the theorem follows.

We now proceed to show that associated with any kernel normal system $A$ there is a congruence $\rho_{A}$ with Ker $\rho_{A}=A$. Let $A$ be Kernel normal system of a regular semigroup $S$. The relation $\rho_{A}$ is defined as follows

$$
\rho_{A}=\left\{(a, b) \in S \times S: x a y \in A_{i} \Leftrightarrow x b y \in A_{i} \forall x, y \in S^{1}, i \in I\right\} \text {. }
$$


Lemma 3.2 Let $A=\left\{A_{i}: i \in I\right\}$ be a Kernel normal system of regular semigroup $S$, and define relation $\rho_{A}$ as above. Then $\rho_{A}$ is a congruence on $S$ with Kernel $A$.

Proof The relation $\rho_{A}$ is clearly an equivalence relation. To show $\rho_{A}$ is a congruence, let $(a, b) \in \rho_{A}$ and $c \in S$. We have $x$ cay $\in A_{i}$ for some $i \in I$ if and only if $x c b y \in A_{i}$ for all choices of $x$ and $y$ in $S^{1}$, since $x c \in S^{1}$. Hence $(c a, c b) \in \rho_{A}$. Similarly, $(a c, b c) \in \rho_{A}$.

To show the Kernel of $\rho_{A}$ is $A$, we have to show that each $A_{i}$ is a $\rho_{A^{-}}$-class. Let $i \in I$. By condition $\mathrm{C}_{2}$, there is an idempotent $e$ in $A_{i}$. Let $a \in e \rho_{A}$. We have to show $a \in A_{i}$. Now $(a, e) \in \rho_{A}$; since $e \in A_{i}$, we have $1 e 1 \in A_{i}$ and so $a=1 a 1 \in A_{i}$. Thus $e \rho_{A} \subseteq A_{i}$.

Conversely, let $b \in A_{i}$. We have to show that $e \rho_{A} b$. Let $x, y \in S^{1}$, and suppose xey $\in A_{j}$. We have that $x A_{i} y \cap A_{j} \neq \varphi$ so that by condition $\mathrm{C}_{3}, x A_{i} y \subseteq A_{\mathrm{j}}$ and $x b y \in A_{\mathrm{j}}$. Similarly, $x$ by $\in A_{j}$ implies that $x e y \in A_{j}$. Thus $e \rho_{A} b$ and $A_{i} \subseteq e \rho_{A}$. It follows that

$\operatorname{Ker} \rho_{A}=A$.

Now we have

Theorem 3.2 Let A be kernel normal system of a regular semigroup S. Then $\rho_{A}$ is the unique congruence on $S$ with $\operatorname{Ker} \rho_{A}=A$.

Proof The existence of $\rho_{A}$ follows from Lemma 3.2, the uniqueness from Theorem 3.1.

Definition 3.1 A regular semigroup in which the idempotents form a subsemigroup that is, a band called an orthodox semigroup.

Theorem 3.3 If S is regular semigroup, then the following statements are equivalent:

a. Sis orthodox,

b. for every $a, b \in S$, if $a^{-1} \in V(a), b^{-1} \in V(b)$, then $b^{-1} a^{-1} \in V(a b)$,

c. if e is idempotent then every inverse of $e$ is idempotents.

As shown, it is possible to characterize the Kernel (Ker) of a congruence $\rho$ on a regular semigroup $\mathrm{S}$ as a set $A=\left\{A_{i}=: i \in I\right\}$ of subsets of $\mathrm{S}$ satisfies the conditions $C_{1}, C_{2}$ and $C_{3}$.

In 1954 Preston [6] gave easier conditions in the case of inverse semigroups. Later Meakin [4,5] gave corresponding conditions to extend Preston's theory from inverse semigroups to orthodox semigroups.

Let $A=\left\{A_{i}: i \in I\right\}$ be a set of subsets of an orthodox semigroup. Then Meakin's conditions defined as follows

D $) A_{i} \cap A_{j}=\varphi$ if $i \neq j$,

$D_{2}$ ) each $A_{i}$ contains an idempotent and each idempotent of $S$ is contained in some $A_{i}$, 
$\left.\mathrm{D}_{3}\right)$ if $a \in A=\mathrm{U}_{i \in I} A_{i}$, then $V(a) \subseteq A$,

$\mathrm{D}_{4}$ ) for each $a \in S$, for each inverse $a^{-1}$ of $a$ and for each $i \in I$, there exists $j \in I$ such that $\quad a A_{i} a^{-1} \subseteq A_{j}$,

$\mathrm{D}_{5}$ ) for each pair $i, j \in I$, there exists $k \in I$ such that $A_{i} A_{j} \subseteq A_{k}$,

$\left.\mathrm{D}_{6}\right)$ if $a, b a \in A_{i}$ and $b^{-1} a, b^{-1} b \in A_{j}$ for some $b^{-1} \in V(a)$ and $i, j \in I$, then $b \in A_{i}$.

We remark here that in $[5,3]$ Meakin called the set $A$ which satisfies these conditions a Kernel normal system. He also gave extra conditions as follows:

$\mathrm{D}_{6}$ ) if $a, b a, b^{-1} b \in A_{i}$ for some $b^{-1} \in V(b)$, then $b \in A_{i}$,

$\left.\mathrm{D}_{7}\right)$ if $\mathrm{e}, a e \in A_{i}$ and $a^{-1} e, a a^{-1} \in A_{j}$ for some elements $e \in E_{x} a^{-1} \in V(a)$ and $i, j \in I$, then $a \in A_{i}$.

It is easy to show that these conditions follow from the previous ons.

For a collection $A$ of subsets satisfying $\mathrm{D}_{1} \ldots \mathrm{D}_{6}$ we define the relation $\sim$ on $\mathrm{U}_{i \in I} A_{i}$ by $a \sim b$ if and only if $a, b$ belong to $A_{i}$ for some $i \in I$.

We now introduce $\rho_{A}$ as we did previously in the regular semigroup

$\rho_{A}=\left\{(a, b) \in S \times S: \exists a^{-1}\right.$ of $a, b^{-1}$ of $\left.b \exists b a^{-1} \sim a a^{-1}, b^{-1} a \sim b^{-1}\right\}$.

Note that, if $\mathrm{A}$ is a set of subsets of an orthodox semigroup $\mathrm{S}$ satisfying $\mathrm{D}_{1} \ldots \ldots . . \mathrm{D}_{6}$, and if $a, b \in S$, then a necessary and sufficient condition for $(a, b) \in \rho_{A}$ is that $b a^{-1} \sim a a^{-1}, b^{-1} a \sim b^{-1}, a^{-1} b \sim a^{-1} a$, and $a b^{-1} \sim b b^{-1}$

For any $a^{-1} \in V(a)$ and $b^{-1} \in V(b)$.

Lemma 3.3 Let $S$ be an orthodox semigroup, $\rho$ be a congruence on $S$ and let $\operatorname{Ker} \rho=A=\left\{A_{i}: i \in I\right\}$. Put $A=U A_{i}$ and $V(A)=U_{a \in A} V(a)$. Then $V(A) \subseteq A$.

Theorem 3.4 Let $S$ be an orthodox semigroup. Then the system $A=\left\{A_{i}, i \in I\right\}$ of subsets of $S$ satisfies conditions $C_{1}, C_{2}$ and $C_{3}$ if and only if it satisfies conditions $D_{1} \ldots . D_{6}$.

Proof Suppose that $A=\left\{A_{i} ; i \in I\right\}$ satisfies the conditions $\mathrm{C}_{1}, \mathrm{C}_{2}$ and $\mathrm{C}_{3}$. Conditions $\mathrm{D}_{1}, \mathrm{D}_{2}$ are the same as $\mathrm{C}_{1}, \mathrm{C}_{2}$. By Theorem 3.2 we have that a congruence $\rho$ exists such that $A=\operatorname{Ker} \rho$, and so condition $\mathrm{D}_{3}$ follows from Lemma 3.3. To prove $\mathrm{D}_{4}$, let $a \in S$ and $a^{-1} \in V(a)$. By $\mathrm{C}_{2}$, there exists $e \in A_{i}$ for some $e \in E$, and since $a e a^{-1} \in E$, we have by $\mathrm{C}_{2}$, aea $a^{-1} \in A_{i}$ for some $j \in I$. Then $a A_{i} a^{-1} \cap A_{j} \neq \varphi$.

Now by $\mathrm{C}_{3}, a A_{i} a^{-1} \subseteq A_{j}$. The condition $\mathrm{D}_{5}$ follows from the fact that each $A_{i}$ is a congruence class. To prove condition $\mathrm{K}_{6}$, let $a, b a \in A_{i}$ and $a^{-1} a, b^{-1} b \in A_{j}$ for some $b^{-1} \in V(b)$ and $i, j \in I$. Then $a \rho b a$, and $b^{-1} a \rho b^{-1} b$, and so as $\rho$ is a congruence, $b b^{-1} a \rho b b^{-1} b a=b a$. Then $a \rho b a \rho b b^{-1} a \rho b b^{-1} b=b$ as $b^{-1} a \rho b^{-1} b$. Hence $a \rho b$, and since $a \in A_{i}$ we have $b \in A_{i}$. 
Conversely, suppose that $A$ satisfies $D_{1}, \ldots, D_{6}$. We need to show that $A$ satisfies condition $C_{3}$. Suppose that $x A_{i} y \cap A_{j} \neq \varphi$, and set $a \in A_{i}$, be such that $x a y \in A_{j}$. Let $c \in A_{i}$, we have to show that $x c y \in A_{j}$. Let $x^{-1} \in V(x), y^{-1} \in V(y)$ and $a^{-1} \in V(a)$. Then $y^{-1} a^{-1} x^{-1} \in V(x a y)$ and $y^{-1} c^{-1} x^{-1} \in V(x c y)$. Since $y y^{-1}$ is idempotent, $y y^{-1} \in A_{k}$ for some $k$. By $\mathrm{D}_{3}, a^{-1} \in A_{h}$ for some $h$ and so by $\mathrm{D}_{5}, c y y^{-1} a^{-1}$, ayy $y^{-1} a^{-1} \in A_{m}$ for some $m \in I$. Now by $\mathrm{D}_{4}$, we have $x c y y^{-1} a^{-1} x^{-1} \sim x a y y^{-1} a^{-1} x^{-1}$.

Similarly,

$$
y^{-1} c^{-1} x^{-1} x a y \sim y^{-1} c^{-1} x^{-1} x c y .
$$

It follows that $x a y \rho_{A} x c y$.

Now let $e \in A_{j}$. We aim to show that if $u \in A_{j}$ and $u \rho_{A} v$, then $v \in A_{j}$. Now we have that if $u^{-1}$ is any inverse of $u$, then $u^{-1} \in A_{k}$ for some $k \in I_{s}$ by $\mathrm{D}_{3}$. Then by $\mathrm{D}_{5}, e u^{-1} \sim u u^{-1}$.

Furthermore, it follows from $\mathrm{K}_{2}$ and $\mathrm{K}_{5}$ that each $A_{i}$ is a subsemigroup and so

$$
\text { ue } \sim e u \sim e .
$$

Since $u \rho_{A} v$, we have

$$
v u^{-1} \sim u u^{-1} \text { and } v^{-1} u \sim v^{-1} v \text {. }
$$

Also

$$
u^{-1} v \sim u^{-1} u \text { and } u v^{-1} \sim v v^{-1} .
$$

Now we show that $e \rho_{A} v$. We first prove that $e \sim e v^{-1} v e$ and $v v^{-1} \sim v e v^{-1}$. Using $\mathrm{D}_{5}$ we have $e\left(v^{-1} v\right) e \sim u\left(v^{-1} v\right) e=(u)\left(u^{-1} u\right)\left(v^{-1} v\right) e \sim u\left(u^{-1} v\right)\left(v^{-1} v\right) e=u\left(u^{-1} v\right) e \sim u\left(u^{-1} u\right) e=u e \sim e e$
$=e$.

Hence $e\left(v^{-1} v\right) e \sim e$. Also, using $\mathrm{D}_{5}$, we have

$$
\begin{gathered}
v e v^{-1}=v\left(v^{-1} v\right) e v^{-1} \sim v v^{-1} u e v^{-1}=v\left[v^{-1} u u^{-1} u e\right] v^{-1} \sim v\left[v^{-1} u u^{-1} u\right] v^{-1} \\
=v\left[v^{-1} u\right] v^{-1} \sim v v^{-1} v v^{-1}=v v^{-1}
\end{gathered}
$$

so that

$$
v v^{-1} \sim v e v^{-1}
$$

Similarly, we can get

$$
v^{-1} v \sim v^{-1} e v
$$

Now set $a=u v^{-1} v u^{-1}$ e. Using $\mathrm{D}_{5}$ we have

$$
a \sim v v^{-1} v u^{-1} e=v u^{-1} e \sim u u^{-1} e \sim u u^{-1} u e=u e \sim e e=e .
$$

Hence $a \sim e$. Now set $b=v e$ and $b^{-1}=e v^{-1}$ so that

$$
b a=\left(v e u v^{-1}\right)\left(v u^{-1}\right) e \sim\left(v e v^{-1}\right)\left(v u^{-1}\right) e \sim\left(v v^{-1}\right)\left(v u^{-1}\right) e=v u^{-1} e \sim u u^{-1} u e=u e \sim e .
$$

Hence $b a \sim e$. Also $b^{-1} b=e v^{-1} v e \sim e$. Hence we have $a \sim b a \sim b^{-1} b$, and so by $\mathrm{D}_{6}$, we have that

$$
b=v e \sim e .
$$


Again, by putting $a=e u^{-1} v v^{-1} u$ and by $\mathrm{D}_{5}$ we have $a \sim u u^{-1} u v^{-1} u \sim u v^{-1} u \sim e v^{-1} v$. Put $b=e v$ and $b^{-1}=v^{-1} e$. Then $b a=e v e u^{-1} v v^{-1} u \sim e e u^{-1} u v^{-1} u \sim u u^{-1} u v^{-1} u=u v^{-1} u \sim u v^{-1} v \sim e v^{-1} v$. Also

$$
\begin{aligned}
& b^{-1} b=v^{-1} e e v \sim v^{-1} e v \sim v^{-1} v \text { and } \\
& b^{-1} a=v^{-1} e e u^{-1} v v^{-1} u \sim v^{-1} e u^{-1} v v^{-1} u \sim v^{-1} u u^{-1} v v^{-1} v \sim v^{-1} u v^{-1} v \sim v^{-1} v v^{-1} v \sim v^{-1} v .
\end{aligned}
$$

Hence we have $a \sim b a$ and $b^{-1} a \sim b^{-1} b$ and so by $\mathrm{D}_{6}$ we have $b=e v \sim e v^{-1} v$, so that $v^{-1} e \in A_{k}$ for some $k \in I$. Now we have

$$
\begin{aligned}
v^{-1} e=\left(v^{-1} e\right) & (e) \sim\left(v^{-1} e\right)(e u) \\
& =\left(v^{-1} e\right)(e u)\left(u^{-1} u\right) \sim v^{-1}\left(e\left(u u^{-1}\right)\right) v \sim\left(v^{-1} u\right)\left(u^{-1} v\right) \sim\left(v^{-1} u\right)\left(v^{-1} u\right) \\
& =v^{-1} u \sim v^{-1} v
\end{aligned}
$$

and hence

$$
v^{-1} e \sim v^{-1} v \text {. }
$$

Now $e \rho_{A} v$. Also we have $e \sim v e \in A_{j}$ as $\epsilon A_{j}$, and $v^{-1} e \sim v^{-1} v_{x}$ so that by $\mathrm{D}_{7}$ we have $v \in A_{j}$. Now we have proved that if $u \in A_{j}$ and $u \rho_{A} v$, then $v \in A_{j}$, and because we have $x a y \rho_{A} x c y$ and $x c y \in A_{j}$, we have that $x c y \in A_{j}$ which implies that $x A_{i} y \subseteq A_{j}$ and $\mathrm{C}_{3}$ is satisfied.

\section{Congruences on inverse semigroups}

Congruences on inverse semigroups via the (kernel-trace) method introduced by Scheiblich in 1974. In this section we discuss the congruences on inverse semigroups by using the technique of Kernel normal systems.

In [7], Preston showed that it is possible to characterize the Kernel of a congruence on an inverse semigroup as a set $A=\left\{A_{i}: i \in I\right\}$ of subsets of $\mathrm{S}$ satisfying the following conditions:-

$\mathrm{I}_{1}$ ) each $A_{i}$ is an inverse subsemigroup of $S_{x}$

I $) A_{i} \cap A_{j}=\varphi$ if $i \neq j$,

$\mathrm{I}_{3}$ ) each idempotent in $S$ is contained in some element of $A$,

$\mathrm{I}_{4}$ ) for each $a \in S$ and $i \in I, a^{-1} A_{i} a \subseteq A_{j}$ for some $\mathrm{j}$, and we can write $j=i a_{\text {, }}$ so that $a^{-1} A_{i} a \subseteq A_{i a}$, $\left.\mathrm{I}_{5}\right)$ if $, a b, b b^{-1} \in A_{\bar{i}}$, then $b \in A_{\bar{i}}$.

We now introduce the congruence $\sigma_{A}$ associated with such a set $A$ of subsets of $S$ as follows

$$
\sigma_{A}=\left\{(a, b) \in S \times S: a a^{-1}, b b^{-1}, a b^{-1} \in A_{i} \text { for some } i \epsilon I\right\} \text {. }
$$

Our aim in this section is to show that if $A$ is a collection of subsets of an inverse semigroup $S$ satisfying conditions $\mathrm{I}_{1}, \ldots \mathrm{I}_{5}$, then $A$ is a kernel normal system and $\sigma_{A}=\rho_{A}$. We begin by proving the following lemma. 
Lemma 4.1 For any $i, j$ in $I$ there exists $k$ in $I$ such that $A_{i} A_{j} \subseteq A_{k}$.

Proof For each $i \in I$ we denote $E\left(A_{i}\right)$ by $E_{i}$ and first we prove that given $i, j \in I$ we have $E_{i} E_{j} \subseteq E_{k}$, for some $k \in I$. If $e \in E_{i}$ and $f \in E_{j}$, we know that $e f$ is idempotent, so ef $\in E_{k}$ for some $k$ by $\mathrm{I}_{3}$. Let $g \in E_{i}, h \in E_{j}$. We have to show that $g h \in E_{k}$. From $\mathrm{I}_{4}$, we have $e A_{j} e \subseteq A_{l}$ for some $l$.

But $e f=e f e \in A_{k p}$ so eff $A_{l} \cap A_{k}$ which implies that $A_{l}=A_{k}$ by $\mathrm{I}_{1}$, that is, $e A_{j} e \subseteq A_{k}$ Now eh $=$ ehe $\epsilon e A_{j} e \subseteq A_{k j}$ so $e h \in A_{k}$ and $e h=$ heh $\in h A_{i} h \subseteq A_{m}$ for some $m$, that is, eh $\in A_{k} \cap A_{m}$ which implies that $A_{k}=A_{m}$ by $\mathrm{I}_{1}$ and so $h A_{i} h \subseteq A_{k}$. Hence $g h=h g h \in A_{k}$ and so $E_{i} E_{j} \subseteq E_{k}$.

Now let $a \in A_{i}, b \in A_{j}$ and put $x=a^{-1} a b a^{-1} a$ and $y=a^{-1} a b$. Note that

$$
a^{-1} a E_{j} a^{-1} a \subseteq E_{i} E_{j} E_{i} \subseteq E_{k s}
$$

so that $a^{-1} a A_{j} a^{-1} a \cap A_{k} \neq \varphi$. But by $\mathrm{I}_{4}, a^{-1} a A_{j} a^{-1} a \subseteq A_{p}$ for some $p$ and so $A_{k} \cap A_{p} \neq \varphi$. Hence $p=k$ and

$$
a^{-1} a A_{j} a^{-1} a \subseteq A_{k}
$$

In particular, $x \in A_{k}$ and so $x x^{-1} \in A_{k}$. Put $u=x x^{-1}$. Now

$u y=x x^{-1} y=a^{-1} a b a^{-1} a a^{-1} a b^{-1} a^{-1} a a^{-1} a b=a^{-1} a\left(b a^{-1} a b^{-1}\right) a^{-1} a b=$ $a a^{-1} a a^{-1} a b a^{-1} a b^{-1} b=a^{-1} a b b^{-1} b a^{-1} a=a^{-1} a b a^{-1} a=x \in A_{k}$

Also

$$
y y^{-1}=a^{-1} a b b^{-1} a^{-1} a \in a^{-1} a A_{j} a^{-1} a
$$

and so $y y^{-1} \in A_{k}$. We now have $u, u y, y y^{-1} \in A_{k}$ and so by $\mathrm{I}_{5}$, we get $y \in A_{k}$.

Using $E_{j} E_{i} \subseteq E_{k}$ and $a^{-1} \in A_{i}$, a similar argument shows that $b b^{-1} a^{-1} \in A_{k}$ and so by $\mathrm{I}_{1}$, we have

$a b b^{-1}=\left(b b^{-1} a^{-1}\right)^{-1} \in A_{k}$. Again by $I_{1}$, we have $\left(a b b^{-1}\right)\left(a^{-1} a b\right) \in A_{k}$. But

$$
\left(a b b^{-1}\right)\left(a^{-1} a b\right)=a\left(b b^{-1}\right)\left(a^{-1} a\right) b=a\left(a^{-1} a\right)\left(b b^{-1}\right) b=a b
$$

and so $a b \in A_{k}$. Thus $A_{i} A_{j} \subseteq A_{k}$ and the lemma is proved.

The following corollary is an immediate consequence of the lemma.

Corollary 4.1 Let $A=\left\{A_{i}: i \in I\right\}$ be a collection of subsets of an inverse semigroup $S$ and let $A=\mathrm{U}_{i \in I} A_{i}$. If A satisfies conditions $I_{1}, \ldots, I_{5}$ then $A$ is an inverse subsemigroup of $S$.

Theorem 4.1 Let $S$ be an inverse semigroup and let $A=\left\{A_{i}: i \in I\right\}$ be a collection of subsets of $S$.

Then $A$ is a Kernel normal system if and only if A satisfies the conditions $I_{1}, \ldots, I_{5}$.

Proof Suppose that $A$ satisfies conditions $\mathrm{D}_{1}, \ldots, \mathrm{D}_{6}$. Then by Theorem 3.4, $A$ is Kernel normal system, and by Theorem 3.2, there is a unique congruence $\rho$ with $\operatorname{Ker} \rho=A$. Now conditions $\mathrm{I}_{2}$ and $\mathrm{I}_{4}$ are the same as conditions $\mathrm{D}_{1}$ and $\mathrm{D}_{4}$. Condition $\mathrm{I}_{3}$ follows trivially from condition $\mathrm{D}_{2}$. 
To prove condition $\mathrm{I}_{1}$, let $a, b \in A_{i}$ for some $i \in I$. Also we have $e \in A_{i}$ for some $e \in E$ by $\mathrm{D}_{2}$. Then $a \rho e$ and $b \rho e$ which implies that $a b \rho e e=e$ as $\rho$ is a congruence. Hence $a b \in A_{i}$ and so $\mathrm{A}_{\mathrm{i}}$ is a subsemigroup. Also $A_{\bar{i}}$ is inverse since if $a \in A_{\bar{i}}$ and $e \in A_{i}$ for $e \in E$, then $a \rho e$ which implies that $a^{-1} \rho e^{-1}=e$ as $S$ is inverse. Hence $a^{-1} \in A_{i}$ for $i \in I$.

To prove condition $\mathrm{I}_{5}$, suppose that $a, a b, b b^{-1} \in A_{i}$. Then $a \rho b b^{-1}$, and $a b \rho b b^{-1} b=b$ as $\rho$ is a congruence. Thus $a b \rho b$ which implies that $b \in A_{i}$ as $a b \in A_{i}$.

Conversely, suppose that $A$ satisfies the conditions $\mathrm{I}_{1}, \ldots, \mathrm{I}_{5}$. We prove that $A$ satisfies conditions $\mathrm{D}_{1}, \ldots \mathrm{D}_{6}$. Conditions $\mathrm{K}_{1}$ and $\mathrm{K}_{4}$ are the same as conditions $\mathrm{I}_{2}$ and $\mathrm{I}_{4}$. Condition $\mathrm{D}_{2}$ is immediate from conditions $\mathrm{I}_{1}$ and $\mathrm{I}_{3}$, and condition $\mathrm{D}_{3}$ follows from $\mathrm{I}_{1}$. Condition $\mathrm{D}_{5}$ follows from Lemma 4.1.

To prove condition $\mathrm{D}_{6}$ suppose that $a, b a \in A_{i}$ and $b^{-1} a, b^{-1} b \in A_{j}$ for some $i, j \in I$. We have to show that $b \in A_{i}$. To use $\mathrm{I}_{5}$, we need that $a b, b b^{-1} \in A_{i}$. Set $y=b b^{-1}$ and $x=b a$ so that $x \in A_{i}$. By $\mathrm{I}_{3}$, we have $y \in A_{k}$ for some $k$ and so $x y \in A_{i} A_{k}$. Now $A_{i} A_{k} \subseteq A_{p}$ for some $p$ by Lemma 4.1 , and so $x y \in A_{p}$. Since $a a^{-1} \in A_{i}$ we also have $y a a^{-1}=a a^{-1} y \in A_{i} A_{k}$ and consequently, $A_{k} A_{i} \subseteq A_{p}$. Since $b a \in A_{i}$, we now have

$$
b a=b b^{-1} b a=y b a \in A_{p} .
$$

Hence $i=p$ and $x y \in A_{i}$.

Also $y=y^{2}=b b^{-1} b b^{-1} \in b A_{j} b^{-1}$ and by $\mathrm{I}_{4}, b A_{j} b^{-1} \subseteq A_{r}$ for some $r$. But $y \in A_{k}$ so that $b A_{j} b^{-1} \subseteq A_{k}$. Hence $b b^{-1} a b^{-1} \in A_{k}$ and so

$$
a a^{-1} b b^{-1} a b^{-1}=b b^{-1} a a^{-1} a b^{-1}=b b^{-1} a b^{-1} \in A_{k^{*}}
$$

Now $a a^{-1} b b^{-1} a b^{-1} \in A_{i} b A_{j} b^{-} \subseteq A_{i} A_{k} \subseteq A_{i}$ and so $b b^{-1} a b^{-1} \in A_{i}$. Thus $i=k$ and so $y y^{-1}=y \in A_{i}$. Thus $x, x y, y y^{-1} \in A_{i}$. Using $\mathrm{I}_{5}$ we have $y=b b^{-1} \in A_{i}$.

Now put $z=b a a^{-1} b^{-1}$ and note that $z \in A_{i}$ since $b a \in A_{i}$ and $A_{i}$ is an inverse subsemigroup of $\mathrm{S}$. Also

$$
z b=b a a^{-1} b^{-1} b=b b^{-1} b a a^{-1}=b a a^{-1} \in A_{i}
$$

Since $a, b a \in A_{i}$ and $A_{i}$ is an inverse subsemigroup of $S$. We have already seen that $b b^{-1} \in A_{i}$ so that now we have, $z, z b, b b^{-1} \in A_{i}$ and hence by $\mathrm{I}_{5}, b \in A_{i}$. Therefore $A$ is Kernel normal system.

Preston gave some alternative conditions which are

$\mathrm{I}_{4}$ ) if $a a^{-1} b b^{-1} a b^{-1} \in A_{i}$, then for any $j \in I$, there exists $k \in I$ such that $a A_{j} a^{-1} \subseteq A_{k}$ and $a A_{j} b^{-1} \subseteq A_{k}$,

$\mathrm{I}_{5}$,) if $a, a b^{-1}, b b^{-1} \in A_{\tilde{i}}$, then $b \in A_{\tilde{i}}$.

In fact, conditions $I_{1}, I_{2}, I_{3}, I_{4}$ and $I_{5}$ are equivalent to conditions $I_{1}, \ldots . I_{5}$. To prove this we need to prove the following lemma.

Lemma 4.2 Let $A=\left\{A_{i}: i \in I\right\}$ be a collection of subsets of an inverse semigroup $S$ satisfying conditions $I_{1, \ldots, I_{5}}$. If $a b^{-1} \in A_{\tilde{i}}$, then $A_{i a}=A_{i b}$. 
Proof By $\mathrm{I}_{1}, A_{\tilde{i}}$ is subsemigroup of $S$ so that $b a^{-1} \in A_{i} \quad$ and also $\left(a b^{-1}\right)\left(b a^{-1}\right) \in A_{\tilde{i}}$ and $\left(b a^{-1}\right)\left(a b^{-1}\right) \in A_{i}$. Hence

$$
a^{-1} a b^{-1} b=a^{-1} a a^{-1} a b^{-1} b=a^{-1} a b^{-1} b a^{-1} a=a^{-1}\left(a b^{-1} b a^{-1}\right) a \epsilon A_{i a} .
$$

Also

$$
a^{-1} a b^{-1} b=b^{-1} b a^{-1} a=b^{-1} b b^{-1} b a^{-1} a=b^{-1} b a^{-1} a b^{-1} b=b^{-1}\left(b a^{-1} a b^{-1}\right) b \in A_{i b}
$$

Hence $A_{i a} \cap A_{i b} \neq \varphi$ and by $\mathrm{I}_{2}, A_{i a}=A_{i b}$.

Proposition 4.1 Let $A=\left\{A_{\bar{i}}: i \in I\right\}$ be a collection of subsets of inverse semigroup $S$. Then A satisfies conditions $I_{1}, \ldots, I_{5}$ if and only if it satisfies conditions $I_{1}, I_{2}, I_{3}, I_{4^{\prime}}, I_{5}$.

Proof Suppose first that $A$ satisfies conditions $\mathrm{I}_{1}, \ldots, \mathrm{I}_{5}$. To show that condition $\mathrm{I}_{4}$ holds, let $i, j \in I$ and let $a, b \in S$ be such that $a a^{-1}, b b^{-1}, a b^{-1}$ are all in $A_{\mathrm{i}}$. By condition $\mathrm{I}_{4}$ we have $a A_{\mathrm{j}} a^{-1} \subseteq A_{\mathrm{k}}$ for some $k \in I$. Note that $a a^{-1} \in A_{i}$ and so

$$
a A_{j} a^{-1}=a A_{j} a^{-1} a a^{-1} \subseteq A_{k} A_{i}
$$

so that $A_{k} \cap A_{k} A_{i} \neq \varphi$. It follows from Lemma 4.1 that $A_{k} A_{i} \subseteq A_{k}$.

Since $A_{j}$ is an inverse subsemigroup of $S$, it contains an idempotent, say $e$.Then

$$
a e b^{-1}=a a^{-1} a e b^{-1}=\left(a e a^{-1}\right)\left(a b^{-1}\right) \varepsilon A_{k} A_{i} \subseteq A_{k}
$$

so that $A_{k} \cap a A_{j} b \neq \varphi$. By Theorem 3.4, $A$ is a Kernel normal system and hence by definition, $A$ satisfies condition $\mathrm{C}_{3}$. Thus $a A_{j} b^{-1} \subseteq A_{k}$ as required.

We now prove condition $\mathrm{I}_{5}$. Now $A$ satisfies condition $\mathrm{I}_{1}, \ldots, \mathrm{I}_{5}$ and so by Theorem $3.4, A$ is Kernel normal system and by Theorem3.2, there is a unique congruence $\rho$ with $\operatorname{Ker} \rho=A$. Let $a, b \in S$ be such that $a, a b^{-1}, b b^{-1} \in A_{i}$. We have to show that $b \in A_{i}$. Now we have $a \rho b b^{-1}$, so that $a b \rho b b^{-1} b=b$ as $\rho$ is a congruence. Also $a \rho a b^{-1}$, so that

$$
a b \rho a b^{-1} b=a a^{-1} a b^{-1} b=a b^{-1} b a^{-1} a .
$$

But $\left(b a^{-1}\right) a \rho b b^{-1}$, since $A_{i}$ is an inverse subsemigroup of $S$ and $b a^{-1}, a \in A_{i}$. Then we have $a b \rho a b^{-1} b a^{-1} a \rho a b^{-1} b b^{-1}=a b^{-1}$.

Since $a b^{-1} \in A_{i}$, it follows $a b \in A_{i}$ which implies that $b \in A_{i}$ by $\mathrm{I}_{5}$. Conversely, suppose that $A$ satisfies $\mathrm{I}_{1}, \mathrm{I}_{2}$, $\mathrm{I}_{3}, \mathrm{I}_{4}$ and $\mathrm{I}_{5}$. We need to show that $A$ satisfies conditions $\mathrm{I}_{4}$ and $\mathrm{I}_{5}$. Let $a \in S$ and $i \in I$. Since $A_{i}$ is an inverse subsemigroup of $S$, it follows that $a^{-1} a \in A_{i}$. Using $\mathrm{I}_{4}$ with $a^{-1}$ in place of $a$ and $b$ we deduce that

$$
a^{-1} A_{i}\left(a^{-1}\right)^{-1} \subseteq A_{k}
$$

for some $k$. But $\left(a^{-1}\right)^{-1}=a$ and so $\mathrm{I}_{4}$ is satisfied.

To show that condition $\mathrm{I}_{5}$ holds, let $a, b \in S$ be such that $a, a b, b b^{-1} \in A_{i}$. By $\mathrm{I}_{1}, A_{i}$ is an inverse subsemigroup and so $(a b) b^{-1} \in A_{i}$. Therefore, putting $x=a b$ we have $x, x b^{-1}, b b^{-1} \in A_{i}$. By $\mathrm{I}_{5}$, this gives $b \in A_{i}$. 


\section{Conclusions}

Congruences on inverse semigroups by using the technique of kernel normal systems developed in this paper and several characterizations of Kernel normal systems are shown to be equivalent.

\section{References}

[1] Clifford, A. H. and Preston, G. B. (1961). The Algebraic theory of semigroups. American Mathemathical Society vol.II.

[2] Howie, John M. (1995). Fundamentals of semigroup theory. London Mathematical Society.

[3] Meakin, John (1970). Congruences on regular semigroups. Semigroup Forum, 1(1970), 232-235.

[4] Meakin, John (1971). Congruences on orthodox semigroups I. J. Austral Math. Soc. 12 (1971), 323-341.

[5] Meakin, John (1972). Congruences on orthodox semigroups II. J. Austral Math. Soc. 13 (1972), 259-266.

[6] Petrich, M. (1978). Congruences on inverse semigroups. J. Algebra, 55 231-256.

[7] Preston, G. B.(1954). Inverse semigroups. J. London Math. Soc., 29 (1954), 96-403. 